lichst weitgehende Erfassung des natürlichen Kapitals angesehen wird, da viele Ressourcen und ökologische Leistungen direkt damit in Zusammenhang stehen. Die eigentliche Bewertung ist somit schon mit der Methode festgelegt. Hintergrund der Bewertung ist somit zum einen das Wissen derjenigen, die die Methode entwickelt haben, zum anderen deren Werthaltung. Die Größe des Fußabdrucks hängt von der Bevölkerungsanzahl, dem materiellen Lebensstandard, der verwendeten Technologie und der Produktivität der Ökosysteme ab. Benötigt eine Region mehr Fläche zur Erhaltung ihres Konsums, als ihr selbst auf ihrem Gebiet zur Verfügung steht, dann muß sie aus anderen Regionen „Flächennutzungen“ importieren, wobei zu berücksichtigen ist, daß die ökologischen Fußabdrücke sich nicht überlappen können: Einmal angeeignete Carrying Capacity steht zumindest in der selben Periode - keinem anderen mehr zur Verfügung (vgl. Wackernagel, Rees 1996, 1997).

\section{Literatur}

- Ankele, K./ Steinfeld, M. (1996): Ökobilanz für typische Ytong-Produktanwendungen. Berlin: IÖW-Schriftenreihe

- Ankele, K./ Rubik, F.. (1997): Charakterisierung und Anwendungsmöglichkeiten ausgewählter Bewertungsverfahren. Berlin: I0̈W-Schriftenreihe (Erscheint im Herbst)

- Hanley, N./ Spash, C. (1993): Cost-Benefit-Analysis and the Environment. Aldershot

- Rubik, F./ Teichert, V. (1997): Ökologische Produktpolitik. Stuttgart

- Wackernagel, M./ Rees, W. (1996): Our Ecological

Footprint. Reducing Human Impact on the Earth

- Weimann, J. (1996): Monetarisierungsverfahren aus der Sicht der ökonomischen Theorie. In: Linckh, G. u. a.

( $\mathrm{Hg}$. ): Nachhaltige Land- und Forstwirtschaft. Berlin, 415 440

- Tanner, C./ Foppa, K. (1996): Umweltwahrnehmung, Umweltbewußtsein und Umweltverhalten, in: Diekmann,

A., Jaeger, C. C. (Hg.): Umweltsoziologie. Opladen, 245 271

\section{Die Autorlnnen}

Kathrin Ankele ist wissenschaftliche Mitarbeiterin im Forschungsbereich ökologische Unternehmenspoliiik, Jürgen Meyerhoff im Bereich Umweltpolitik und Umwelı̈̈konomie am Institut für ökologische Wirtschaftsforschung in Berlin.

Kontakt: IÖW, Giesebrechtstr. 13, 10629 Berlin, Tel. (030) $884594-0$

Präferenzen für Umweltgüter in der ökonomischen Theorie

\title{
Hintertür im Gefangenen-Dilemma
}

\author{
Menschen verhalten sich in zwei verschiedenen Entscheidungssituationen, für \\ die es die gleiche eindeutige Lösung gibt, trołzdem unterschiedlich, wenn ihnen \\ die Situationen unterschiedlich präsentiert werden. Im Dilemma der Übernut- \\ zung von Umweltgütern kann eine bestimmte Präsentation des Problems die \\ Akteure deshalb zu stärkerer Kooperation bewegen. Der Staat könnte diesen \\ positiven „Framing-Effekt" nułzen, ohne direkt regulierend in das Verhalten \\ der einzelnen einzugreifen.
}

$\mathrm{D}$ Von Joachim Weimann e meisten Ökonomen dürften darin übereinstimmen, daß das methodische Fundament der ökonomischen Theorie der methodologische Individualismus ist. Das bedeutet jedoch keineswegs, daß alle die gleiche methodische Position einnehmen, denn was konkret unter dem methodologischen Individualismus zu verstehen ist, darüber gibt es durchaus unterschiedliche Auffassungen. Unstrittig ist allerdings die Art und Weise, in der Ökonomen mit individuellen Präferenzen umzugehen pflegen.

Präferenzen - oder ersatzweise deren Abbildung durch eine Nutzenfunktion (1) - spielen in der ökonomischen Theorie eine ambivalente Rolle. Ausgehend von einem anthropozentrischen Weltbild gebietet der methodologische Individualismus auf der einen Seite, daß allein der Nutzen, den Menschen aus Dingen ziehen, Dingen einen Wert geben kann. Daß etwas Menschen Nutzen stiftet, ist eine notwendige Bedingung dafür, daß es einen Wert haben kann. Insofern ist die Präferenz für ein Gut neben der Knappheit (die für das ökonomische Kardinalproblem sorgt) die zweite zentrale Kategorie der gesamten Wirtschaftswissenschaft. Auf der anderen Seite wird die ökonomische Theorie von zwei grundlegenden Voraussetzungen geprägt, die den Umgang mit Präferenzen in äußerst restriktiver Weise regeln:

1. Präferenzen sind als gegeben $\mathrm{zu}$ betrachten, das heißt, die Entstehung von Präferenzen oder ihr Wandel ist nicht Gegenstand ökonomischer Theorien.

2. Präferenzen sind private Information, das heißt, allein das Individuum weiß (und kann wissen), welche Präferenzen es tatsächlich besitzt.
Natürlich steht der Grundsatz (1.) in einer engen Beziehung zu der Hypothese (2.). Niemand hat das Recht, einem anderen vorzuschreiben, was ihm nützt. Dies verbietet jeden „diktatorischen“ Umgang mit Präferenzen und gibt der Subjektivität der Bewertung den Vorzug. Um dennoch wissenschaftliche Sätze ableiten zu können, die bekanntlich intersubjektiv überprüfbar sein sollten, bleibt kaum eine andere Möglichkeit, als Aussagen zu machen, die für gegebene, aber beliebige Präferenzen gelten.

Der Umgang mit individuellen Präferenzen ist darïber hinaus durch ein weiteres methodisches Prinzip geprägt, das in der ökonomischen Theorie eine zentrale Rolle spielt: die Annahme rationalen Verhaltens der Akteure. Das Rationalitätspostulat gestattet es, theoretische Schlüsse zu ziehen, weil die Logik als universelles Instrument des Entscheidens eingesetzt werden kann. Man benötigt keine ausdifferenzierte Verhaltenstheorie, um Aussagen über individuelles Verhalten zu machen, solange vorausgesetzt werden kann, daß das Verhalten das Ergebnis eines rationalen Optimierungskalküls ist. Es macht die unübertroffene Stärke der ökonomischen Entscheidungstheorie aus, mit dem Rationalitätspostulat das gesamte Instrumentarium der Mathematik für die Analyse von Entscheidungen nutzbar gemacht zu haben.

Damit läßt sich der Umgang, den die ökonomische Theorie mit Präferenzen pflegt, wie folgt beschreiben: Präferenzen sind private Informationen, die als gegeben, aber beliebig anzusehen sind und beziiglich derer sich Individuen konsistent verhalten (2). Im normativen Kontext hat sich die ökonomische Methodik als ausgesprochen erfolgreich erwiesen. Mit Hilfe von Modellen, die auf der Basis des Rationalitätspostulates abgeleitet werden, gelingt es vielfach, komplexe Zusammenhänge zu strukturieren und versteh- 
bar zu machen, die ohne eine ausdifferenzierte formale Theorie nicht verstanden werden könnten. Modelle schaffen selbst dann wertvolle Bezugspunkte des Denkens, wenn sie kontrafaktische Gegenentwürfe zur Realität produzieren. Auch wenn es oft nicht gelingt, Realität korrekt abzubilden, erleichtern Modelle das Verständnis der Realität, weil sie theoretisch wohlfundierte Wenn-dann-Aussagen ableiten, das heißt, die Voraussetzungen, unter denen solche Aussagen Gültigkeit beanspruchen können, klar benennen. Die Verwendung formaler Methoden ermöglicht dies alles immer in Verbindung mit einem intersubjektiven Konsistenznachweis der theoretischen Aussage und erleichtert den Nachweis der Wissenschaftlichkeit erheblich.

\section{- Präferenzen für Umweltgüter werden strategisch verborgen}

Im Hinblick auf Umweltgüter hat die normative Theorie zu einigen eindeutigen und wichtigen Befunden gefuihrt, die sich insbesondere aus deren Charakter als öffentlichem Gut ergeben. Unter der Voraussetzung, daß Individuen vom Konsum dieser Güter nicht ausgeschlossen werden können, werden sie bei rationalem Verhalten ihren Informationsvorsprung, der sich aus dem privaten Charakter der Präferenzen ergibt, strategisch nutzen und nicht bereit sein, ihre wahren Präferenzen für Umweltguiter zu offenbaren. Daraus folgt (3):

- Die dezentrale Allokation von Umweltgütern führt zu einem ineffizienten Resultat.

- Der soziale Planer ist nicht in der Lage, dieses Marktversagen zu heilen, weil er nicht erwarten kann, in den Besitz der dafür notwendigen Präferenzinformation zu gelangen.

Liegt damit das Thema Präferenzen für Umweltgüter nicht außerhalb der ökonomischen Zuständigkeit? Wenn man es bei der normativen Theorie belassen würde, wäre dem ganz sicher so, denn im normativen Kontext von der Voraussetzung beliebiger, gegebener Präferenzen abzurücken wäre nur schwer mit dem Anspruch

\section{Der Autor}

Dr. Joachim Weimann ist Professor für Volkswirtschaftslehre an der Otto-von-Guericke-Universität in Magdeburg.

Kontakt: Fakultär für Wirtschaftswissenschaft, Lehrstuhl für Volkswirtschaftslehre, Posifach 4120 , 39016 Magdeburg, Tel. (0391) 67-185 47 auf Wissenschaftlichkeit zu vereinbaren. Aber daneben existiert ein legitimes wissenschaftliches Interesse an der möglichst korrekten Beschreibung und der Analyse beobachtbaren Verhaltens - mithin an positiver Theorie. In diesem Kontext gibt es nun aber einen zentralen Befund, der der Voraussetzung gegebener Präferenzen zuwiderläuft.

\section{Widerspruch zur Theorie des rationalen Verhaltens}

Eine unmittelbare Implikation des Rationalitätspostulates besteht darin, daß Individuen dann, wenn eine eindeutige rationale Lösung ihres Optimierungsproblems existiert, sie diese auch wählen werden und daß sie folglich in zwei unterschiedlichen Entscheidungssituationen, in denen die gleiche eindeutige Lösung existiert, sich auch gleich verhalten werden. Der zentrale Befund, der hier von Bedeutung ist, lautet jedoch: Dies ist nicht der Fall, das heißt, Menschen können in unterschiedlich präsentierten Situationen mit gleicher rationaler Lösung unterschiedliche Verhaltensweisen zeigen. R. Selten (1990) hat schon früh in diesem Zusammenhang von Präsentationseffekten gesprochen, der heute gebräuchliche Terminus technicus für dieses Phänomen ist „Framing-Effekt"“.

Der Nachweis solcher Effekte ist der experimentellen Wirtschaftsforschung zu verdanken. Sie konnte unter Laborbedingungen zeigen, daß die Art und Weise, wie Menschen sich verhalten, sehr stark davon abhängt, wie sie die Entscheidungssituation wahrnehmen. Der vielleicht bekannteste Fall eines Framing-Effekts ist die sogenannte „Präferenzumkehr“ bei der Bewertung von Lotterien. Die Präferenz zwischen zwei Lotterien kann sich umkehren, wenn einmal eine Bewertung in Form eines Reservationspreises abgefragt und anschließend eine Wahl zwischen den bewerteten Lotterien herbeigefuihrt wird: Vielfach wird die Lotterie gewählt, der zuvor der niedrigere Reservationspreis zugeordnet wurde. Diese Inkonsistenz ist mit keiner bekannten Theorie rationalen Verhaltens unter Unsicherheit vereinbar.

\section{Altruistische Strafegie}

Es existieren aber auch wichtige Beispiele für Framing-Effekte, die unmittelbar im Zusammenhang mit den Präferenzen für Umweltgüter stehen. Das gewissermaßen paradigmatische Modell zur Beschreibung der Ursachen für
Umweltprobleme ist das sogenannte Gefangenen-Dilemma (GD). In seiner üblichen Formulierung läßt es sich in Normalform als $2 \times 2$ Matrix angeben:

\section{Abb. 1: Gefangenen-Dilemma (GD) - Normalform}

\begin{tabular}{|ccc|} 
& $\begin{array}{c}\text { Spieler 2 } \\
\text { C }\end{array}$ & $\begin{array}{c}\text { Spieler 2 } \\
\text { D }\end{array}$ \\
\hline Spieler 1 (C) & 12,12 & 0,18 \\
\hline Spieler 1 (D) & 18,00 & 6,60 \\
\hline
\end{tabular}

Beide Spieler haben in diesem Beispiel zwei Strategien C und D. Die Matrix zeigt die Auszahlungen der Spieler bei den vier möglichen Strategiekombinationen. Die Werte sind so gewählt, daß sich das bekannte Resultat einstellt: Das einzige Gleichgewicht in diesem Spiel ist ein Nash-Gleichgewicht in dominanten Strategien (4), in dem beide Spieler D wählen; dieses Gleichgewicht ist ineffizient, weil mit $(C, C)$ eine Alternative existiert, die beide Spieler strikt besser stellen würde.

Um das Umweltproblem anhand des GD zu erläutern, reicht es, wenn man sich vorstellt, daß C eine "umweltgerechte" Verhaltensweise ist, die Kosten bei dem einzelnen Akteur verursacht, beiden Spielern aber Vorteile verschafft, und D eine auf den Schaden des jeweils anderen Akteurs setzende Strategie, bei der die eigenen Kosten für umweltgerechtes Verhalten eingespart werden können.

Wie verhalten sich nun Menschen, wenn man sie mit einem solchen Dilemma konfrontiert? Dieser Frage ist in zahlreichen psychologischen und ökonomischen Experimenten nachgegangen worden (5). Bereits 1970 verglich D. G. Pruitt die Kooperationsraten in dem oben beschriebenen GD mit denen in einem modifizierten Spiel, das sich von der üblichen GD-Formulierung ausschließlich in der Präsentation der Pay-offs unterschied. Die Versuchspersonen mußten wiederum zwischen D und C wählen, aber es wurde ihnen nicht die Normalform des Spiels präsentiert, sondern folgende Auszahlungsregel:

\section{Abb. 2: Gefangenen-Dilemma - unilaterale Formulierung}

\begin{tabular}{|lcc|}
$\begin{array}{l}\text { Wähle selbst } \\
\text { Strategie }\end{array}$ & $\begin{array}{l}\text { Veränderung des } \\
\text { eigenen Payoffs }\end{array}$ & $\begin{array}{l}\text { Veränderung } \\
\text { des Payoffs des } \\
\text { Mitspielers }\end{array}$ \\
\hline C & 0 & 12 \\
\hline D & 6 & 0 \\
\hline
\end{tabular}


Wie man leicht überprüfen kann, reproduziert diese unilaterale Formulierung des Spiels das oben beschriebene $\mathrm{GD}$, das heißt, die strategischen Bedingungen (Strategiemenge, Auszahlungsfunktion) wird durch die Veränderung der Payoff-Darstellung nicht berïhrt, beide Spiele sind strategisch identisch. Dennoch beobachtete Pruitt, daß sich bei der unilateralen Formulierung der Kooperationsanteil deutlich gegenüber der Normalform-Variante erhöhte. Offensichtlich macht der „Frame“ der unilateralen Formulierung deutlich, daß es um die Entscheidung zwischen einer eher altruistischen (C) und einer egoistischen Strategie (D) geht und dieser Frame erzeugt stärkere Kooperationsbereitschaft.

Ein sehr ähnliches Phänomen wurde von J. Andreoni (1995) in einem Öffentlichen-Gut-Experiment beobachtet. Dabei handelt es sich um einen Experimenttyp, der eine Verallgemeinerung des GD untersucht und dabei die Struktur vieler Umweltprobleme noch deutlicher abbildet als das Zwei-Personen-GD. In dem Experiment von Andreoni bildeten je fünf Spieler eine Gruppe. Jeder Spieler wurde mit 60 Spielmarken ausgestattet, die auf zwei Anlagen verteilt werden mußten. Jede Marke, die in die ,private" Anlage investiert wurde, erbrachte einen Ertrag von 1 Cent für den betreffenden Spieler, jede in die öffentliche Anlage investierte Marke führte $\mathrm{zu}$ einer Auszahlung von 1/2 Cent für jeden der fünf Spieler.

Das Spiel wurde jeweils zehnmal in immer neu zusammengesetzten Gruppen wiederholt, und zwar in zwei Varianten, die sich nur durch die Darstellung der Payoffs unterschieden, das heißt, unterschiedliche Frames aufwiesen. In Variante 1 wurde den Spielern mitgeteilt, daß jede Marke, die sie in die öffentliche Anlage investierten, zu einem Ertrag bei den anderen Spielern in Höhe von $1 / 2$ Cent fuihrt. In Variante 2 wurde dagegen betont, daß jede privat investierte Marke die Auszahlung aller Spieler um $1 / 2$ Cent reduziert (6). Während Variante 1 den positiven externen Effekt der Kooperation betont, stellt der Frame der zweiten Variante den negativen externen Effekt unterlassener Kooperation in den Vordergrund. Abbildung 3 gibt die durchschnittlichen Beiträge in die öffentliche Anlage in beiden Varianten wieder.

Das Ergebnis ist eindeutig: Der positive Frame, in dem die Spieler durch Kooperation ,anderen etwas Gutes tun", ist weitaus erfolgreicher als der negative, in dem Kooperation darin besteht,

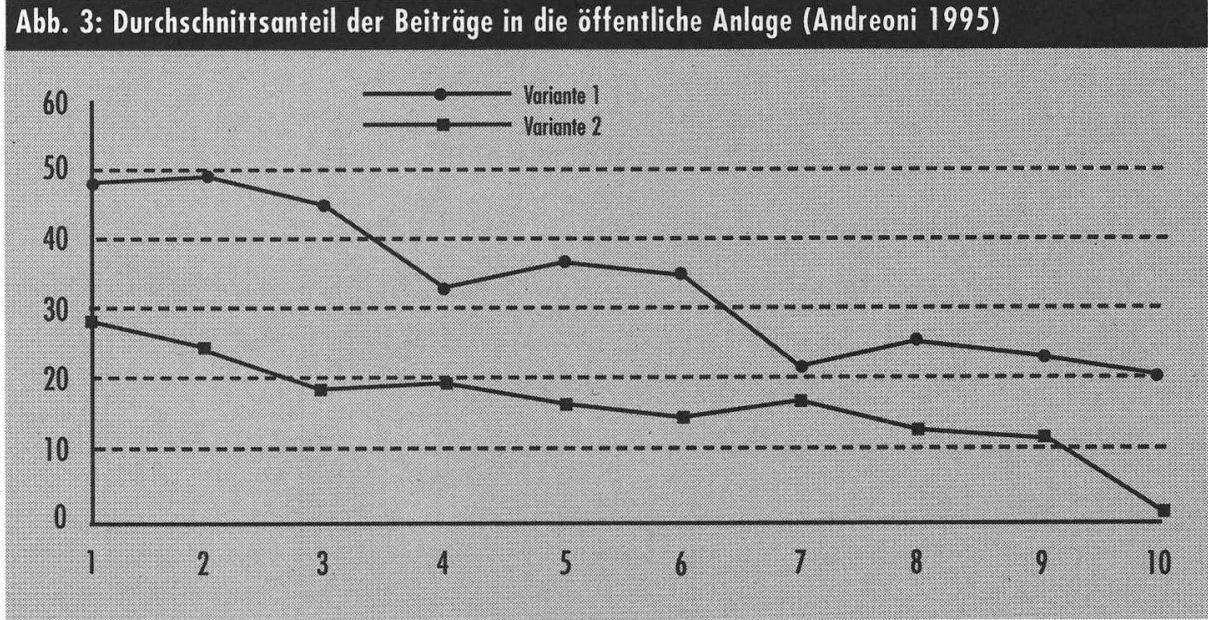

,anderen nichts Schlechtes zu tun“, oder in den Worten Andreonis: „... it must be that people enjoy doing a good deed more than they enjoy not doing a bad deed" (1995).

\section{Rahmen des Umweltverhaltens läßßs sich veröndern}

Warum ist die Erkenntnis, daß der Frame eines sozialen Dilemmas für das Verhalten der darin gefangenen Individuen bedeutsam ist, wichtig? Man muß sich klarmachen, daß es unmöglich ist, Framing-Effekte auszuschließen, weil es unmöglich ist, etwas ohne einen Frame zu präsentieren. Darüber hinaus ist der Frame bedeutsamer sozialer Dilemmata - und mit solchen haben wir es im Falle der Umweltproblematik zu tun - eher eine Variable als eine Konstante. Der Planer ist durchaus in der Lage, die Präsentation des Problems zu beeinflussen und zu verändern. Er verändert damit unter Umständen das Verhalten der Menschen, ohne regulierend einzugreifen und ohne direkten Einfluß auf die Präferenzen der Menschen nehmen zu wollen (etwa im Sinne einer Meritorisierung von Umweltgütern).

Eine solche Framing-Strategie wäre deshalb mit den Grundsätzen des methodologischen Individualismus in jedem Fall vereinbar und würde zugleich die üblichen Nachteile staatlicher Regulierungen vermeiden. Dazu ist es allerdings notwendig, im positiv theoretischen Kontext von der Annahme konstanter Präferenzen bei strikt rationalem Verhalten Abschied zu nehmen.

\section{Anmerkungen}

(1) Man sollte beachten, daß mit dem Begriff "Nutzen" nicht impliziert werden soll, daß es sich bei Präferenzordnungen um eine Abbildung von "Lust und Schmerz", in einem utilitaristischen Sinne handelt. Mit Präferenzen ist lediglich gemeint, daß ein Güterbündel einem anderen vorgezogen wird. Warum dies geschieht, welche Empfindungen damit verbunden sind, bleibt offen.

(2) Zu den Implikationen dieser Vorgehensweise vgl. Weimann 1996, Kapitel 1.

(3) Für eine ausführliche Betrachtung vgl. Weimann 1995a, Teil 1.

(4) Gleichgültig welche Strategie Spieler i wählt, für Spieler $j$ ist es immer die beste Antwort, D zu spielen.

(5) Zu den Resultaten vgl. die Übersichtsartikel von Weimann 1995b oder Ledyard 1995.

(6) Formal sind die beiden Frames wie folgt beschrieben: Es sei $x_{i}$ die private Anlage des i-ten Spielers und gi sein Beitrag in die offentliche Anlage. Dann ist die Auszahlung

im positiven Frame: $\pi_{i}=x_{i}+\frac{1}{2} g_{i}+\frac{1}{2} \sum_{i \neq} g_{i}$

und im negativen Frame $\pi_{i}=x_{i}+\frac{1}{2} g_{i}+\frac{1}{2} \sum_{i \neq i} x_{i}+120$.

\section{Literafur}

- Andreoni, J. (1995): Warm Glow Versus Cold Prickle: The Effects of Positive and Negative Framing on Cooperation in Experiments, Quarterly Journal of Economics, $X_{X}, 2-22$

- Pruitt, D. G. (1970): Motivational Processes in the Decomposed Prisoners Dilemma Game, Journal of Personality and Social Psychology, 14, 227-237

- Ledyard, J. O. (1995): Public Goods: A Survey of Experimental Research, in: Kages, J. H./Roth, A. (eds.): Handbook of Experimental Economics, Princeton, S. 111-181.

- Selten, R. (1990): Bounded Rationality, JITE, 146, 649-658

- Weimann, J. (1995a): Umwelïökonomik. Eine theorieorientierte Einführung, Berlin, Heidelberg et al., 3. Auflage - Weimann, J. (1995b): Freifahrer im Test: Ein Überblick über 20 Jahre Freifahrerexperimente, in: Ökonomie und Gesellschaft, Jahrbuch 12: Soziale Kooperation, 168-241 - Weimann, J. (1996): Wirtschaftspolitik - Allokation und kollektive Entscheidung, Berlin et al 
(c) 20I0 Authors; licensee IÖW and oekom verlag. This is an article distributed under the terms of the Creative Commons Attribution Non-Commercial No Derivates License (http://creativecommons.org/licenses/by-nc-nd/3.o/), which permits unrestricted use, distribution, and reproduction in any medium, provided the original work is properly cited. 